\title{
Interesting QFT Problems Tackled in New Fashion
}

\author{
A. Plastino ${ }^{1,2,3}$, M. C. Rocca ${ }^{1,2,4}$ \\ ${ }^{1}$ Departamento de Física, Universidad Nacional de La Plata, La Plata, Argentine \\ ${ }^{2}$ IFLP-Consejo Nacional de Investigaciones Científicas y Tecnológicas (IFLP-CCT-CONICET)-C. C., La Plata, \\ Argentina \\ ${ }^{3}$ SThAR-EPFL, Lausanne, Switzerland \\ ${ }^{4}$ Departamento de Matemática, Universidad Nacional de La Plata, La Plata, Argentine \\ Email: angeloplastino@gmail.com
}

How to cite thi s paper: Plastino, A. and Rocca, M.C. (2020) Interesting QFT Problems Tackled in New Fashion. Journal of High Energy Physics, Gravitation and Cosmology, 6, 590-608.

https://doi.org/10.4236/jhepgc.2020.64040

Received: July 10, 2020

Accepted: August 14, 2020

Published: August 17, 2020

Copyright (c) 2020 by author(s) and Scientific Resea rch Publishing Inc.

This work is licensed under the Creative Commons Attribution International License (CC BY 4.0).

http://creativecommons.org/licenses/by/4.0/

\section{Open Access}

\section{Abstract}

The Dimensional Regularization technique of Bollini and Giambiagi (BG) [Phys. Lett. B 40, 566 (1972); Il Nuovo Cim. B 12, 20 (1972); Phys. Rev. D 53, 5761 (1996)] cannot be employed for all Schwartz Tempered Distributions Explicitly Lorentz Invariant (STDELI) $\mathcal{S}_{L}^{\prime}$. We lifted such limitation in [J. Phys. Comm. 2 115029 (2018)], which opens new QFT possibilities, centering in the use of STDELI that allows one to obtain a product in a ring with zero divisors. This in turn, overcomes all problems regrading QFT infinities. We provide here three examples of the application of our STDELI-extension to quantum field theory (A) the exact evaluation of an electron's self energy to one loop, (B) the exact evaluation of QED's vacuum polarization, and C) the $\lambda \frac{\phi^{4}}{4 !}$ theory for six dimensions, that is non-renormalizable.

\section{Keywords}

Dimensional Regularization Generalization, Electron Self Energy, Vacuum Polarization, Six-Dimensional Non Renormalizable $\lambda \frac{\phi^{4}}{4 !}$ Theory

\section{Introduction}

The current paradigm in quantum field theory (QFT) asserts that non renormalizable theories are intractable. This is so because an infinite number of counter terms are needed to deal with the infinities that arise in the convolution of two quantum propagators.

A nascent new paradigm, based on Ultrahyperfunctions (UHF), a generalization of the well known Schwartz' distributions, uses the fact 
that quantum propagators can be represented by UHF-distributions. If one does it, then convolutions are always finite and infinities disappear. Non renormalizable theories can then be tackled with no undue effort. This fact is highly unpalatable for workers of the old paradigm that will fight to the death the propagation of the new one.

Thus, this effort is based on quantum field theoretical (QFT) results of the new paradigm, exhaustively discussed with all pertinent details in [1-6]. They revolve around Ultrahyperfunctions theory (UHT), a generalization of Schwartz' distribution theory. Specifically, one uses a particular kind of Schwartz's distributions called explicitly Lorentz invariant ones (STDELI). We will revisit here via STDELI the exact evaluation of an electron's self energy to one loop, the exact evaluation of QED's vacuum polarization, and the $\lambda \frac{\phi^{4}}{4 !}$ theory in six (non renormalizable) dimensions. We use extensively the mathematics of [1-6], without giving all the intermediate results for the sake of saving space. For the sake of completeness, though, some mathematical sketch is given below, which may be skipped.

\subsection{Brief Summary of the Mathematical Results to Be Employed}

This subsection may be omitted at a first reading. Our work revolves around the problem of using a workable version of the product of two distributions (a product in a ring with divisors of zero). This is an old conundrum of hard functional analysis. In quantum field theory (QFT), the problem of evaluating the product of distributions with coincident point singularities is related to the asymptotic behaviour of loop integrals of propagators, becoming thus an obstacle that is to the essence to overcome. We did it in references [1-5].

From a mathematical point of view, practically all definitions of that product of distributions lead to limitations on the set of distributions that can be multiplied by each other to give another distribution of the same type. In fact, Laurent Schwartz himself was unable to define a product of distributions regarded as an algebra, instead of as a ring with divisors of zero.

In references [2-5] it was demonstrated that it is possible to define a general convolution between ultradistributions, introduced by the mathematician J. Sebastiao e Silva and called an ultrahyperfunction [7]. This new convolution yields then an ultrahyperfunction. Therefore, we have a product in a ring with zero divisors. Such a ring is the space of distributions of exponential type, or ultradistributions of exponential type, obtained applying the anti-Fourier transform to the space of tempered ultradistributions or ultradistributions of exponential type. Remember that ultrahyperfunctions are the generalization and extension to the complex plane of the Schwartz' tempered distributions and of the distributions of exponential type [2-5]. That is, the temperate distributions and those of exponential type are a subset of the set of ultrahyperfunctions. One then faces the problem of formulating the convolution between ultradistributions. This is a complex issue, difficult to manage, even if it exhibits the advantage of allowing one to attempting to deal with non-renormalizable QFT's.

Fortunately enough, we have found [1] that a method similar to that used to define the convolution of ultradistributions can also be employed to define the convolution of Lorentz Invariant distributions [8],using a generalization Bollini-Giambiaggi's (BG) dimensional regularization (DR) ( [9-11]) in momentum space [1]. As a consequence, 
ultradistributions need not to be used in our present calculations, which considerably simplifies things. Taking advantage of the regularization technique of [1] one can also work in configuration space. Thus, one is able obtain a convolution of Lorentz Invariant tempered distributions in momentum space and the corresponding product in configuration space [1].

Using the results of [1] we have managed to do the Quantum Field Theory of Einstein's Gravity [12], the Non-Relativistic Quantum Field Theory of Verlinde's Gravity [6] and the Newton's Gravity [13].

It is of the essence to have a clear picture of the consequences of the advances made in reference [1]. As a starting point, remark that DR is one of the most important advances in theoretical physics, being used in several disciplines $[14,15]$. If one adds to it our DRgeneralization [1], the above referred to convolution happens to be both one of STDELI in momentum space and a product in a ring with divisors of zero in configuration space. Our work of [1] can then be used to quantize non-renormalizable QFT's. This could be regarded as a worthwhile achievement.

More to the point, let us emphasize that our [1]-work is concerned with deeper issues than those regarding QFT axiomatics in Euclidean space and QFT renormalization. Reference [1] generalized BG dimensional regularization to all Schwartz tempered, explicitly Lorentz invariant, distributions (STDELI), something that BG were unable to achieve. Permit us to insist on the fact that this would permit one to deal with non-renormalizable QFT's.

A great advantage thereof lies in the fact that we do not have to use counterterms in a renormalization process devoted to eliminate infinities. This is exactly what we would not want to do, since a non-renormalizable theory involves an infinite number of counterterms. The central purpose of our work is to avoid counterterms. In addition, we do not appeal to a simple correlation-functions' convolution (not defined for all STDELI). At the same time, we conserve all extant solutions to the problem of running coupling constants and the renormalization group. The STDELI convolution, once obtained, converts configuration space into a ring with zero-divisors. In it, one has now defined a product between the ring-elements. Thus, any unitary-causal-Lorentz invariant theory quantified in such a manner becomes predictive. The distinction between renormalizable on not-renormalizable QFT's becomes unnecessary now. With our BG generalization, that uses Laurent's expansions in the dimension, all finite constants of the convolutions become completely determined, eliminating arbitrary choices of finite constants. This is tantamount to eliminating all finite renormalizations of the theory. What is the importance of using only that term independent of the dimension in Laurent's expansion? That the result obtained for finite convolutions will coincide with such a term. This fact translates to configuration space the product-operation on a ring with divisors of zero.

\subsection{Present Goals}

We wish here to illustrate the power of our approach with reference to two interesting examples. In such a vein we calculate two convolutions of distributions used in QFT, in connection with the electron self energy and the vacuum polarization in QED. 


\section{Preliminary Materials}

\subsection{The BG-Original Calculation of an Electron Self Energy to One Loop}

The self energy of the electron is defined as

$$
\Sigma(p, \nu)=\frac{e^{2}}{(2 \pi)^{\nu}} \int \gamma_{\mu} \frac{[i \gamma \cdot(p-k)-m]}{\left[(p-k)^{2}+m^{2}\right] k^{2}} \gamma^{\mu} d^{\nu} k .
$$

BG evaluated this integral for the first time in [10]. BG's result, obtained using their definition of DR, is

$$
\begin{gathered}
\Sigma(p, \nu)=\frac{e^{2}}{(4 \pi)^{\frac{\nu}{2}}}\left(m^{2} \rho\right)^{\frac{\nu}{2}-2} \Gamma\left(2-\frac{\nu}{2}\right)\left\{[(i \gamma \cdot p+m)(2-\nu)-2 m] \frac{2}{\nu-2} .\right. \\
F\left(2-\frac{\nu}{2}, \frac{\nu}{2}-1, \frac{\nu}{2} ; 1-\frac{1}{\rho}\right)+[m-(i \gamma \cdot p+m)]\left(\frac{4}{\nu}-2\right) . \\
\left.F\left(2-\frac{\nu}{2}, \frac{\nu}{2}, \frac{\nu}{2}+1 ; 1-\frac{1}{\rho}\right)\right\}
\end{gathered}
$$

where the variable $\rho$ is defined as $\rho=\left(p^{2}+m^{2}\right) / m^{2}$. To obtain the finite part of the self energy, BG decomposed it in the form

$$
\Sigma(p, \nu)=A+(i \gamma \cdot p+m) B+(i \gamma \cdot p+m)^{2} \Sigma_{f}(p, \nu),
$$

where $A, B$, and $\Sigma_{f}(p, \nu)$ were defined as

$$
\begin{gathered}
A=[\Sigma(p, \nu)]_{i \gamma \cdot p+m=0} \\
B=\left[\frac{\Sigma(p, \nu)-A}{i \gamma \cdot p+m}\right]_{i \gamma \cdot p+m=0} \\
\Sigma_{f}(p, \nu)=\left[\frac{\Sigma(p, \nu)-A-(i \gamma \cdot p+m) B}{(i \gamma \cdot p+m)^{2}}\right]_{i \gamma \cdot p+m=0},
\end{gathered}
$$

with $(i \gamma \cdot p+m)^{-1}=(m-i \gamma \cdot p) /\left(p^{2}+m^{2}\right) . \Sigma_{f}(p, \nu)$ thus turns out to be the finite part of the self energy. As a result of these definitions we get

$$
\begin{gathered}
A=-\frac{e^{2} m^{\nu-3}}{(4 \pi)^{\frac{\nu}{2}}} \frac{\nu-1}{\nu-3} \Gamma\left(2-\frac{\nu}{2}\right), \\
B=\frac{A}{m}, \\
\Sigma_{f}(p, \nu)=\frac{e^{2} m^{\nu-4}}{(4 \pi)^{\frac{\nu}{2}}} \Gamma\left(2-\frac{\nu}{2}\right) \cdot\left\{\left[(2-\nu) \frac{m-i \gamma \cdot p}{m^{2} \rho}-4 \frac{m-i \gamma \cdot p}{m^{2} \rho^{2}}+\frac{2}{m \rho}\right] .\right. \\
\frac{2}{\nu-2} F\left(1,2-\frac{\nu}{2}, \frac{\nu}{2} ; 1-\rho\right)+\left[2 \frac{m-i \gamma \cdot p}{m^{2} \rho^{2}}-\frac{m-i \gamma \cdot p}{m^{2} \rho}-\frac{1}{m \rho}\right]\left(\frac{4}{\nu}-2\right) . \\
\left.F\left(1,2-\frac{\nu}{2}, \frac{\nu}{2}+1 ; 1-\rho\right)+\frac{\nu-1}{\nu-3}\left[2 \frac{m-i \gamma \cdot p}{m^{2} \rho^{2}}-\frac{1}{m \rho}+\frac{m-i \gamma \cdot p}{m^{2} \rho}\right]\right\} .
\end{gathered}
$$

It should be noted that $A$ and $B$ are independent of $p$. The three above equations have not been modified till the present time. However, 
and this is a crucial issue, BG's decomposition exhibits an unwanted facet. There are an infinite number of ways to choose $A$ and $B$. The cause is found in products of the gamma function with the hypergeometric function at a pole of the gamma function. We must therefore isolate such pole in proper fashion, so as to avoid this problem. The rigorous way to do this is to use the convolution of Lorentz Invariant distributions obtained in [1]. If this is done straightforwardly, the result obtained turns out to be too involved to be faced numerically, as one faces quite difficult to evaluate functions. These functions arise when we derive the hypergeometric functions (HF) that appear in (2.1.2) with respect to the dimension $\nu$ so as to perform the corresponding Laurent's series expansion, typical of DR. Instead, before HF-deriving as explained above, one must first isolate the gamma function at the pole (from the hypergeometric function) and subsequently tackle the Laurent expansion. This will be illustrated below.

\subsection{Vacuum Polarization}

BG also calculated the vacuum polarization in QED [10]. The integral that defines it is given by

$$
\Pi_{\mu, v}(k, \nu)=\frac{i e^{2}}{(2 \pi)^{\nu}} \operatorname{Tr} \int \gamma_{\mu} \frac{i \gamma \cdot p-m}{p^{2}+m^{2}} \gamma_{v} \frac{i \gamma \cdot(p-k)-m}{(p-k)^{2}+m^{2}} d^{\nu} p .
$$

To evaluate it, the following results should be used

$$
\begin{gathered}
\operatorname{Tr} \gamma_{\mu} \gamma_{v}=d(\nu) \eta_{\mu v}, \\
\operatorname{Tr} \gamma_{\mu} \gamma_{v} \gamma_{\rho} \gamma_{\sigma}=d(\nu)\left(\eta_{\mu v} \eta \rho \sigma-\eta_{\mu \rho} \eta_{v \sigma}+\eta_{\mu \sigma} \eta_{v \rho}\right),
\end{gathered}
$$

where $d(\nu)$ is an analytic function of the dimension $\nu$, which, for $\nu$ a positive integer, matches the number of components of the associated spinor in a $\nu$ dimensional space The result of the integral (2.2.1) is

$$
\begin{aligned}
& \Pi_{\mu v}(k, \nu)= \\
& \frac{e^{2}}{(4 \pi)^{\frac{\nu}{2}}} \frac{d(\nu)}{3} \Gamma\left(2-\frac{\nu}{2}\right) m^{\nu-4}\left(k_{\mu} k_{v}-\eta_{\mu v} k^{2}\right) F\left(2-\frac{\nu}{2}, 2, \frac{5}{2} ;-\frac{k^{2}}{4 m^{2}}\right) .
\end{aligned}
$$

Note that in all terms, except for the first one, one faces the multiplication of the gamma function by a zero of the hypergeometric function. Therefore, the finite part of the vacuum polarization $\Pi_{\mu v F}(k, \nu)$ is given by (note a critical -1 subtraction in the last bracket)

$$
\begin{aligned}
\Pi_{\mu v F}(k, \nu)= & \frac{e^{2}}{(4 \pi)^{\frac{\nu}{2}}} \frac{d(\nu)}{3} \Gamma\left(2-\frac{\nu}{2}\right) m^{\nu-4}\left(k_{\mu} k_{v}-\eta_{\mu v} k^{2}\right) . \\
& {\left[F\left(2-\frac{\nu}{2}, 2, \frac{5}{2} ;-\frac{k^{2}}{4 m^{2}}\right)-1\right], }
\end{aligned}
$$

and the vacuum polarization is cast as

$$
\Pi_{\mu v}(k, \nu)=\frac{e^{2}}{(4 \pi)^{\frac{\nu}{2}}} \frac{d(\nu)}{3} \Gamma\left(2-\frac{\nu}{2}\right) m^{\nu-4}\left(k_{\mu} k_{v}-\eta_{\mu v} k^{2}\right)+\Pi_{\mu v F}(k, \nu)
$$




\section{Simple Preliminary Example to Better Understand Our Procedure}

So as to better understand our approach we will confront now with two simple examples: the self-energy and the vacuum polarization, corresponding to a massless scalar field $\phi$ and a massive scalar field $\psi$ with an interaction given by $g \phi \psi^{2}$

\subsection{The Scalar Field Self Energy}

In this case the self energy is given by:

$$
\Sigma(p, \nu)=\int \frac{d^{\nu} k}{k^{2}\left[(p-k)^{2}+m^{2}\right]}
$$

The result of this integral is: (see [9])

$$
\Sigma(p, \nu)=i \pi^{\frac{\nu}{2}} m^{\nu-4} \Gamma\left(1-\frac{\nu}{2}\right) F\left(1,2-\frac{\nu}{2}, \frac{\nu}{2} ;-\frac{p^{2}}{m^{2}}\right)
$$

This last equation can be re-written in the form:

$$
\Sigma(p, \nu)=\frac{2 i \pi^{\frac{\nu}{2}} m^{\nu-4}}{2-\nu} \Gamma\left(2-\frac{\nu}{2}\right) F\left(1,2-\frac{\nu}{2}, \frac{\nu}{2} ;-\frac{p^{2}}{m^{2}}\right)
$$

We use now the equality:

$$
\begin{gathered}
\Gamma\left(2-\frac{\nu}{2}\right) F\left(1,2-\frac{\nu}{2}, \frac{\nu}{2} ;-\frac{p^{2}}{m^{2}}\right)= \\
\Gamma\left(2-\frac{\nu}{2}\right)-\frac{2}{\nu} \Gamma\left(3-\frac{\nu}{2}\right) F\left(1,3-\frac{\nu}{2}, \frac{\nu}{2}+1 ;-\frac{p^{2}}{m^{2}}\right)
\end{gathered}
$$

With it we obtain:

$$
\begin{gathered}
\Sigma(p, \nu)=\frac{2 i \pi^{\frac{\nu}{2}} m^{\nu-4}}{2-\nu} \Gamma\left(2-\frac{\nu}{2}\right)- \\
\frac{4 i \pi^{\frac{\nu}{2}} m^{\nu-6} p^{2}}{\nu(2-\nu)} \Gamma\left(3-\frac{\nu}{2}\right) F\left(1,3-\frac{\nu}{2}, \frac{\nu}{2}+1 ;-\frac{p^{2}}{m^{2}}\right)
\end{gathered}
$$

The self energy can be then decomposed as:

$$
\Sigma(p, \nu)=A+\Sigma_{f}(p, \nu)
$$

where

$$
A=\frac{2 i \pi^{\frac{\nu}{2}} m^{\nu-4}}{2-\nu} \Gamma\left(2-\frac{\nu}{2}\right)
$$

and

$$
\Sigma_{f}(p, \nu)=-\frac{4 i \pi^{\frac{\nu}{2}} m^{\nu-6} p^{2}}{\nu(2-\nu)} \Gamma\left(3-\frac{\nu}{2}\right) F\left(1,3-\frac{\nu}{2}, \frac{\nu}{2}+1 ;-\frac{p^{2}}{m^{2}}\right)
$$

This is the procedure followed by Bollini and Giambiagi. We are going to proceed with our generalization of dimensional dimensional. For this we are going to develop the self-energy in Laurent's series. Let $f$ be given by:

$$
f(\nu)=\frac{\pi^{\frac{\nu}{2}} m^{\nu-4}}{(2-\nu)}
$$


Its development is:

$$
f(\nu)=-\frac{\pi^{2}}{2}+\frac{\pi^{2}}{2}[1-\ln (\pi)-2 \ln (m)](\nu-4)+\sum_{k=2}^{\infty} b_{k}(\nu-4)^{k}
$$

As a consequence we have:

$$
\begin{gathered}
f(\nu) \Gamma\left(2-\frac{\nu}{2}\right)=\frac{\pi^{2}}{\nu-4}+\frac{\pi^{2}}{2}[\ln (\pi)+2 \ln (m)+C-1](\nu-4)+ \\
\sum_{k=2}^{\infty} d_{k}(\nu-4)^{k}
\end{gathered}
$$

Development of self energy then is:

$$
\begin{aligned}
& \Sigma(p, \nu)=\frac{2 i \pi^{2}}{\nu-4}+i \pi^{2}[\ln (\pi)+2 \ln (m)+C-1+ \\
& \left.\frac{p^{2}}{2 m^{2}} F\left(1,1,3 ;-\frac{p^{2}}{m^{2}}\right)\right]+\sum_{k=1}^{\infty} a_{k}(\nu-4)^{k}
\end{aligned}
$$

We can write again:

$$
\Sigma(p, \nu)=A+\Sigma_{f}(p, \nu)
$$

with

$$
A=\frac{2 i \pi^{2}}{\nu-4}
$$

and

$$
\begin{gathered}
\Sigma_{f}(p, \nu)=i \pi^{2}[\ln (\pi)+2 \ln (m)+C-1+ \\
\left.\frac{p^{2}}{2 m^{2}} F\left(1,1,3 ;-\frac{p^{2}}{m^{2}}\right)\right]+\sum_{k=1}^{\infty} a_{k}(\nu-4)^{k}
\end{gathered}
$$

Therefore the exact value of self energy in four dimensions is:

$$
\begin{gathered}
\Sigma_{P}(p, 4)=\Sigma_{f}(p, 4)=i \pi^{2}[\ln (\pi)+2 \ln (m)+C-1+ \\
\left.\frac{p^{2}}{2 m^{2}} F\left(1,1,3 ;-\frac{p^{2}}{m^{2}}\right)\right]
\end{gathered}
$$

\subsection{The Vacuum Polarization of a Scalar Field}

As an example we consider the vacuum polarization, given by

$$
\Pi(p, \nu)=\int \frac{d^{\nu} k}{\left(k^{2}+m^{2}\right)\left[(p-k)^{2}+m^{2}\right]} .
$$

The result of this integral is (see [9])

$$
\Pi(p, \nu)=i \pi^{\frac{\nu}{2}} m^{\nu-4} \Gamma\left(2-\frac{\nu}{2}\right) F\left(1,2-\frac{\nu}{2}, \frac{3}{2} ;-\frac{p^{2}}{4 m^{2}}\right) .
$$

We use the equality

$$
\Gamma\left(2-\frac{\nu}{2}\right) F\left(1,2-\frac{\nu}{2}, \frac{3}{2} ;-\frac{p^{2}}{4 m^{2}}\right)=
$$




$$
\Gamma\left(2-\frac{\nu}{2}\right)-\frac{p^{2}}{6 m^{2}} \Gamma\left(3-\frac{\nu}{2}\right) F\left(1,3-\frac{\nu}{2}, \frac{5}{2} ;-\frac{p^{2}}{4 m^{2}}\right) .
$$

We have then

$$
\begin{gathered}
\Pi(p, \nu)=i \pi^{\frac{\nu}{2}} m^{\nu-4} \Gamma\left(2-\frac{\nu}{2}\right)- \\
\frac{i \pi^{\frac{\nu}{2}} m^{\nu-6} p^{2}}{6} \Gamma\left(3-\frac{\nu}{2}\right) F\left(1,3-\frac{\nu}{2}, \frac{5}{2} ;-\frac{p^{2}}{4 m^{2}}\right) .
\end{gathered}
$$

We make the decomposition

$$
\Pi(p, \nu)=A+\Pi_{f}(p, \nu) .
$$

Thus, we obtain

$$
A=i \pi^{\frac{\nu}{2}} m^{\nu-4} \Gamma\left(2-\frac{\nu}{2}\right)
$$

and

$$
\Pi_{f}(p, \nu)=-\frac{i \pi^{\frac{\nu}{2}} m^{\nu-6} p^{2}}{6} \Gamma\left(3-\frac{\nu}{2}\right) F\left(1,3-\frac{\nu}{2}, \frac{5}{2} ;-\frac{p^{2}}{4 m^{2}}\right) .
$$

This is the result obtained by BG. We proceed now to generalize it. We define

$$
f(\nu)=\pi^{\frac{\nu}{2}} m^{\nu-4}
$$

As a consequence, we obtain the Laurent expansion

$$
\begin{aligned}
f(\nu) \Gamma\left(2-\frac{\nu}{2}\right)=- & \frac{2 \pi^{2}}{\nu-4}-\pi^{2}[C+2 \ln (m)+\ln (\pi)]+ \\
& \sum_{k=1}^{\infty} b_{k}(\nu-4)^{k} .
\end{aligned}
$$

The expression of the vacuum polarization is now

$$
\begin{gathered}
\Pi(p, \nu)=-\frac{2 i \pi^{2}}{\nu-4}-\pi^{2}[C+2 \ln (m)+\ln (\pi)+ \\
\left.\frac{p^{2}}{6 m^{2}} F\left(1,1, \frac{5}{2} ;-\frac{p^{2}}{4 m^{2}}\right)\right]+\sum_{k=1}^{\infty} a_{k}(\nu-4)^{k} .
\end{gathered}
$$

We can re-write it as

$$
\Pi(p, \nu)=A+\Pi_{f}(p, \nu)
$$

with

$$
A=-\frac{2 i \pi^{2}}{\nu-4}
$$

and

$$
\begin{array}{r}
\Pi_{f}(p, \nu)=-\pi^{2}[C+2 \ln (m)+\ln (\pi)+ \\
\left.\frac{p^{2}}{6 m^{2}} F\left(1,1, \frac{5}{2} ;-\frac{p^{2}}{4 m^{2}}\right)\right]+\sum_{k=1}^{\infty} a_{k}(\nu-4)^{k} .
\end{array}
$$

The exact value for the four dimensional vacuum polarization is then

$$
\Pi_{P}(p, 4)=\Pi_{f}(p, 4)=-\pi^{2}[C+2 \ln (m)+\ln (\pi)+
$$




$$
\left.\frac{p^{2}}{6 m^{2}} F\left(1,1, \frac{5}{2} ;-\frac{p^{2}}{4 m^{2}}\right)\right] .
$$

As a second example we give the calculation of the self-energy of a well-known QFT model, in the renormalizable case $\nu=4$ and in the non-renormalizable case $\nu=6$.

\section{The Massless Case for $\frac{\lambda}{4 !} \phi^{4}$ Model}

\subsection{The $\nu$-Dimensional Self-Energy}

As it is well known, in Minkowski's space the second order self energy is defined as (all remaining terms, to second order, vanish using our method)

$$
\Sigma(\rho)=(\rho-i 0)^{-1} *(\rho-i 0)^{-1} *(\rho-i 0)^{-1},
$$

where $\rho=k_{1}^{2}+k_{2}^{2}+k_{3}^{2}+\ldots .+k_{\nu-1}^{2}-k_{0}^{2}$.

Going beyond the mere definition (4.1.1), we will actually evaluate $\Sigma$ now using our methodology. In $\nu$ dimensions we have

$$
\Sigma(\rho, \nu)=\left[(\rho-i 0)^{-1} *(\rho-i 0)^{-1} *(\rho-i 0)^{-1}\right]_{\nu} .
$$

We are thus confronted with the convolution of three massless Feynman's propagators. In facing such problem, we take into account that

$$
\mathcal{F}^{-1}\left\{f_{1} * f_{2} * f_{3}\right\}=(2 \pi)^{2 \nu} \hat{f}_{1} \hat{f}_{2} \hat{f}_{3} .
$$

According to reference [16], we this have

$$
\mathcal{F}^{-1}\left\{(\rho-i 0)^{-1}\right\}=\frac{e^{\frac{i \pi}{2}}}{(2 \pi)^{\nu}} 2^{\nu-2} \pi^{\frac{\nu}{2}} \Gamma\left(\frac{\nu}{2}-1\right)(x+i 0)^{1-\frac{\nu}{2}},
$$

and accordingly,

$$
\begin{gathered}
\mathcal{F}^{-1}\left\{(\rho-i 0)^{-1} *(\rho-i 0)^{-1} *(\rho-i 0)^{-1}\right\}= \\
\frac{e^{\frac{i 3 \pi}{2}}}{(2 \pi)^{\nu}} 2^{3 \nu-6} \pi^{\frac{3 \nu}{2}}\left[\Gamma\left(\frac{\nu}{2}-1\right)\right]^{3}(x+i 0)^{3-\frac{3 \nu}{2}} .
\end{gathered}
$$

Using again reference [16] we face now

$$
\begin{gathered}
\mathcal{F}\left\{(x+i 0)^{3-\frac{3 \nu}{2}}\right\}= \\
\frac{e^{-\frac{i \pi}{2}}}{\Gamma\left(\frac{3 \nu}{2}-3\right)} 2^{6-2 \nu} \pi^{\frac{\nu}{2}} \Gamma(3-\nu)(\rho-i 0)^{\nu-3}
\end{gathered}
$$

with which we can write for $\Sigma(\rho, \nu)$

$$
\begin{gathered}
\Sigma(\rho, \nu)=\left[(\rho-i 0)^{-1} *(\rho-i 0)^{-1} *(\rho-i 0)^{-1}\right]_{\nu}= \\
\frac{e^{i \pi}}{(3-\nu) \Gamma\left(\frac{3 \nu}{2}-3\right)} \pi^{\nu}\left[\Gamma\left(\frac{\nu}{2}-1\right)\right]^{3} \Gamma(4-\nu)(\rho-i 0)^{\nu-3},
\end{gathered}
$$

which successfully completes our task. 


\subsection{A Confirmation of the Validity of the Above Result}

To such an effect, we proceed now to the traditional technique for evaluating $\Sigma$ via the well known Feynman's integrals and parameters. Thus, we have

$$
\begin{gathered}
\Sigma(k)=(\rho-i 0)^{-1} *(\rho-i 0)^{-1} *(\rho-i 0)^{-1}= \\
\int \frac{d^{4} p d^{4} q}{\left(p^{2}-i 0\right)\left(q^{2}-i 0\right)\left[(p+q-k)^{2}-i 0\right]},
\end{gathered}
$$

so that, in $\nu$ dimensions

$$
\begin{gathered}
\Sigma(k, \nu)=\left[(\rho-i 0)^{-1} *(\rho-i 0)^{-1} *(\rho-i 0)^{-1}\right]_{\nu}= \\
\int \frac{d^{\nu} p d^{\nu} q}{\left(p^{2}-i 0\right)\left(q^{2}-i 0\right)\left[(p+q-k)^{2}-i 0\right]} .
\end{gathered}
$$

After performing the corresponding Wick rotations we get then

$$
-\int \frac{d^{\nu} p d^{\nu} q}{p^{2} q^{2}(p+q-k)^{2}}
$$

Let us evaluate first

$$
\Sigma_{1}(k, \nu)=\int \frac{d^{\nu} p}{p^{2}(p+q-k)^{2}} .
$$

We define here $k-q=k_{1}$ and then

$$
\Sigma_{1}\left(k_{1}, \nu\right)=\int \frac{d^{\nu} p}{p^{2}\left(p-k_{1}\right)^{2}} .
$$

We now use Feynman's parameters and obtain

$$
\Sigma_{1}\left(k_{1}, \nu\right)=i \int_{0}^{1} \int \frac{d^{\nu} p d x}{\left[\left(p-k_{1} x\right)^{2}+a\right]^{2}}
$$

where

$$
a=k_{1}^{2} x(1-x) .
$$

Change variables by setting $u=p-k x$ and write

$$
\Sigma_{1}\left(k_{1}, \nu\right)=\int_{0}^{1} \int \frac{d^{\nu} u d x}{\left[u^{2}+a\right]^{2}} .
$$

We evaluate the integral over $u$ using [17] and have

$$
\int \frac{\left(u^{2}\right)^{n}}{\left(u^{2}+a\right)^{m}} d^{\nu} u=\frac{\pi^{\frac{\nu}{2}}}{\Gamma\left(\frac{\nu}{2}\right)} \frac{\Gamma\left(\frac{\nu}{2}+n\right) \Gamma\left(m-n-\frac{\nu}{2}\right)}{\Gamma(m) a^{m-n-\frac{\nu}{2}}},
$$

which leads to:

$$
\Sigma_{1}\left(k_{1}, \nu\right)=i \pi^{\frac{\nu}{2}} \gamma\left(2-\frac{\nu}{2}\right) k_{1}^{\nu-4} \int_{0}^{1} x^{\frac{\nu}{2}-2}(1-x)^{\frac{\nu}{2}-2} d x .
$$


Using [17] once again leads to

$$
\int_{0}^{1} x^{\frac{\nu}{2}-2}(1-x)^{\frac{\nu}{2}-2} d x=\frac{2}{\nu-2} F\left(2-\frac{\nu}{2}, \frac{\nu}{2}-1, \frac{\nu}{2} ; 1\right) .
$$

Also from [17] one finds

$$
F\left(2-\frac{\nu}{2}, \frac{\nu}{2}-1, \frac{\nu}{2} ; 1\right)=\frac{\Gamma\left(\frac{\nu}{2}\right) \Gamma\left(\frac{\nu}{2}-1\right)}{\Gamma(\nu-2)},
$$

so that

$$
\Sigma_{1}\left(k_{1}, \nu\right)=\frac{1}{\Gamma(\nu-2)} \pi^{\frac{\nu}{2}}\left[\Gamma\left(\frac{\nu}{2}-1\right)\right]^{2} \Gamma\left(2-\frac{\nu}{2}\right) k_{1}^{\nu-4} .
$$

Using this result, we have for the self-energy:

$$
\begin{gathered}
\Sigma(k, \nu)=-\frac{1}{\Gamma(\nu-2)} \pi^{\frac{\nu}{2}}\left[\Gamma\left(\frac{\nu}{2}-1\right)\right]^{2} \Gamma\left(2-\frac{\nu}{2}\right) \times \\
\int \frac{d^{\nu} q}{q^{2}\left[(k-q)^{2}\right]^{2-\frac{\nu}{2}}} .
\end{gathered}
$$

We now turn our attention to Feynman's generalized parameters:

$$
\frac{1}{A^{\alpha} B^{\beta}}=\frac{\Gamma(\alpha+\beta)}{\Gamma(\alpha) \Gamma(\beta)} \int_{0}^{1} \frac{x^{\alpha-1}(1-x)^{\beta-1} d x}{[A x+B(1-x)]^{\alpha+\beta}}
$$

The self-energy thus has the expression

$$
\begin{gathered}
\Sigma(k, \nu)=-\frac{1}{\Gamma(\nu-2)} \pi^{\frac{\nu}{2}}\left[\Gamma\left(\frac{\nu}{2}-1\right)\right]^{2} \Gamma\left(3-\frac{\nu}{2}\right) \times \\
\int_{0}^{1} x^{1-\frac{\nu}{2}} \int \frac{d^{\nu} q}{\left[(k-q)^{2} x+q^{2}(1-x)\right]^{3-\frac{\nu}{2}}},
\end{gathered}
$$

or, equivalently:

$$
\begin{aligned}
\Sigma(k, \nu)= & -\frac{1}{\Gamma(\nu-2)} \pi^{\frac{\nu}{2}}\left[\Gamma\left(\frac{\nu}{2}-1\right)\right]^{2} \Gamma\left(3-\frac{\nu}{2}\right) \times \\
& \int_{0}^{1} x^{1-\frac{\nu}{2}} d x \int \frac{d^{\nu} q}{\left[(q-k x)^{2}+a\right]^{3-\frac{\nu}{2}}}
\end{aligned}
$$

We define now $a=k^{2} x(1-x), u=q-k x$, and then the last integral can be easily evaluated as

$$
\int \frac{d^{\nu} u}{\left[\left(u^{2}+a\right]^{3-\frac{\nu}{2}}\right.}=\frac{\pi^{\frac{\nu}{2}}}{\Gamma\left(\frac{\nu}{2}\right)} a^{\frac{\nu}{2}-3} B\left(\frac{\nu}{2}, 3-\nu\right) .
$$

Thus, we have for the self-energy

$$
\begin{gathered}
\Sigma(k, \nu)=-\frac{1}{\Gamma(\nu-2)} \pi^{\nu}\left[\Gamma\left(\frac{\nu}{2}-1\right)\right]^{2} \Gamma(3-\nu) k^{2 \nu-6} \times \\
\int_{0}^{1} x^{\frac{\nu}{2}-2}(1-x)^{\nu-3} d x .
\end{gathered}
$$


Finally, we obtain

$$
\Sigma(k, \nu)=-\frac{1}{\Gamma\left(\frac{3 \nu}{2}-3\right)} \pi^{\nu}\left[\Gamma\left(\frac{\nu}{2}-1\right)\right]^{3} \Gamma(3-\nu) k^{2 \nu-6} .
$$

Returning now to Minkowski's space, we have the following expression for the $\nu$-dimensional self-energy

$$
\Sigma(\rho, \nu)=-\frac{1}{\Gamma\left(\frac{3 \nu}{2}-3\right)} \pi^{\nu}\left[\Gamma\left(\frac{\nu}{2}-1\right)\right]^{3} \Gamma(3-\nu)(\rho-i 0)^{\nu-3}
$$

We will next give the dimension $\nu$ fixed values.

\subsection{The Four-Dimensional Self-Energy}

This is a known problem, that we discuss here only for didactic purposes. In four dimensions the theory $\lambda \frac{\phi^{4}}{4 !}$ is renormalizable.

As the reader should expect, we expand $\Sigma(\nu)$ in Laurent series around the pole at $\nu=4[1-6,12,13]$ and find

$\Sigma(\rho, \nu)=\frac{\pi^{4} \rho}{2(4-\nu)}-\frac{\pi^{4} \rho}{2}\left[\ln (\rho-i 0)+\ln \pi+C-\frac{13}{4}\right]+\sum_{m=1}^{\infty} a_{m}(\nu-4)^{m}$.

The term independent of $\nu-4$ in our expansion yields the selfenergy $[1-6,12,13]$ :

$$
\Sigma(\rho)=-\frac{\pi^{4} \rho}{2}\left[\ln (\rho-i 0)+\ln \pi+C-\frac{13}{4}\right] .
$$

\subsection{Successfully Dealing with the Non Renormalizable Case of 6 Dimensions}

We pose ourselves now a critical test, by showing that we can deal with a yet unsolved instance without undue hardship. When $\nu=6$, the $\lambda \frac{\phi^{4}}{4 !}$ theory is non-renormalizable. Thus, if we appeal to the BG technique, we can not proceed as we did above. The achievement that we will boast about now is that our present Laurent based approach remains undaunted by these difficulties. This will be appreciated below.

Our Laurent expansion is

$$
\begin{aligned}
\Sigma(\rho, \nu) & =\frac{\pi^{6} \rho^{3}}{6 !(6-\nu)}-\frac{\pi^{6} \rho^{3}}{6 !}[\ln (\rho-i 0) \\
& +\ln \pi+C-\frac{187}{60}+\sum_{m=1}^{\infty} a_{m}(\nu-6)^{m}
\end{aligned}
$$

The term independent of $\nu-6$ in our expansion yields the selfenergy $[1-6,12,13]$

$$
\Sigma(\rho)=-\frac{\pi^{6} \rho^{3}}{6 !}\left[\ln (\rho-i 0)+\ln \pi+C-\frac{187}{60}\right]
$$

As promised, no undue hardship was involved. Non renormalizable QFT theories can be easily dealt with by appeal to our techniques of $[1-6,12,13]$. 


\section{Our Exact Results}

\subsection{The Electron Self Energy to One Loop}

To evaluate self energy we use the following equalities

$$
\begin{aligned}
& F\left(2-\frac{\nu}{2}, \frac{\nu}{2}-1, \frac{\nu}{2} ; 1-\frac{1}{\rho}\right)=\rho^{2-\frac{\nu}{2}} F\left(1,2-\frac{\nu}{2}, \frac{\nu}{2} ; 1-\rho\right), \\
& F\left(2-\frac{\nu}{2}, \frac{\nu}{2}, \frac{\nu}{2}+1 ; 1-\frac{1}{\rho}\right)=\rho^{2-\frac{\nu}{2}} F\left(1,2-\frac{\nu}{2}, \frac{\nu}{2}+1 ; 1-\rho\right) .
\end{aligned}
$$

We can write $(2.1 .2)$ in the form

$$
\begin{gathered}
\Sigma(p, \nu)=\frac{e^{2}}{(4 \pi)^{\frac{\nu}{2}}} m^{\nu-4} \Gamma\left(2-\frac{\nu}{2}\right)\left\{[(i \gamma \cdot p+m)(2-\nu)-2 m] \frac{2}{\nu-2} .\right. \\
F\left(1,2-\frac{\nu}{2}, \frac{\nu}{2} ; 1-\rho\right)+[m-(i \gamma \cdot p+m)]\left(\frac{4}{\nu}-2\right) . \\
\left.F\left(1,2-\frac{\nu}{2}, \frac{\nu}{2}+1 ; 1-\rho\right)\right\} .
\end{gathered}
$$

Using the following relations, we can isolate the gamma function at the pole

$$
\begin{gathered}
\Gamma\left(2-\frac{\nu}{2}\right) F\left(1,2-\frac{\nu}{2}, \frac{\nu}{2} ; 1-\rho\right)= \\
\Gamma\left(2-\frac{\nu}{2}\right)+\frac{2(1-\rho)}{\nu} \Gamma\left(3-\frac{\nu}{2}\right) F\left(1,3-\frac{\nu}{2}, \frac{\nu}{2}+1 ; 1-\rho\right), \\
\Gamma\left(2-\frac{\nu}{2}\right) F\left(1,2-\frac{\nu}{2}, \frac{\nu}{2}+1 ; 1-\rho\right)= \\
\Gamma\left(2-\frac{\nu}{2}\right)+\frac{2(1-\rho)}{\nu+2} \Gamma\left(3-\frac{\nu}{2}\right) F\left(1,3-\frac{\nu}{2}, \frac{\nu}{2}+2 ; 1-\rho\right) .
\end{gathered}
$$

Replacing these results in (5.1.3) we obtain

$$
\begin{aligned}
& \Sigma(p, \nu)=\frac{e^{2}}{(4 \pi)^{\frac{\nu}{2}}} m^{\nu-4}\left\{\left\{[(i \gamma \cdot p+m)(2-\nu)-2 m] \frac{2}{\nu-2}+\right.\right. \\
& \left.[m-(i \gamma \cdot p+m)] \frac{4-2 \nu}{\nu}\right\} \Gamma\left(2-\frac{\nu}{2}\right)+ \\
& \left\{[(i \gamma \cdot p+m)(2-\nu)-2 m] \frac{4(1-\rho)}{\nu(\nu-2)} .\right. \\
& F\left(1,3-\frac{\nu}{2}, \frac{\nu}{2}+1 ; 1-\rho\right)+[m-(i \gamma \cdot p+m)] \frac{(8-4 \nu)(1-\rho)}{\nu(\nu+2)} \text {. } \\
& \left.\left.F\left(1,3-\frac{\nu}{2}, \frac{\nu}{2}+2 ; 1-\rho\right)\right\} \Gamma\left(3-\frac{\nu}{2}\right)\right\}
\end{aligned}
$$

or equivalently

$$
\begin{aligned}
\Sigma(p, \nu)= & \frac{e^{2}}{(4 \pi)^{\frac{\nu}{2}}} m^{\nu-4}\left\{\left\{\left(\frac{4}{\nu-2}-\frac{2 \nu-4}{\nu}\right) m-\right.\right. \\
& \left.\frac{4}{\nu}(i \gamma \cdot p+m)\right\} \Gamma\left(2-\frac{\nu}{2}\right)+
\end{aligned}
$$




$$
\begin{gathered}
\left\{[(i \gamma \cdot p+m)(2-\nu)-2 m] \frac{4(1-\rho)}{\nu(\nu-2)} .\right. \\
F\left(1,3-\frac{\nu}{2}, \frac{\nu}{2}+1 ; 1-\rho\right)+[m-(i \gamma \cdot p+m)] \frac{(8-4 \nu)(1-\rho)}{\nu(\nu+2)} . \\
\left.\left.F\left(1,3-\frac{\nu}{2}, \frac{\nu}{2}+2 ; 1-\rho\right)\right\} \Gamma\left(3-\frac{\nu}{2}\right)\right\}
\end{gathered}
$$

Note that $1-\rho=-p^{2} / m^{2}$. We can decompose the self energy in the form

$$
\Sigma(p, \nu)=A+B(i \gamma \cdot p+m)+\Sigma_{f}(p, \nu),
$$

where $A, B$, and $\Sigma_{f}(p, \nu)$ are given by

$$
\begin{gathered}
A=\frac{e^{2}}{(4 \pi)^{\frac{\nu}{2}}} m^{\nu-3}\left(\frac{4}{\nu-2}-\frac{2 \nu-4}{\nu}\right) \Gamma\left(2-\frac{\nu}{2}\right), \\
B=\frac{e^{2}}{(4 \pi)^{\frac{\nu}{2}}} m^{\nu-4} \frac{4}{\nu} \Gamma\left(2-\frac{\nu}{2}\right), \\
\Sigma_{f}(p, \nu)=\frac{e^{2}}{(4 \pi)^{\frac{\nu}{2}}} m^{\nu-4}\left\{[(i \gamma \cdot p+m)(2-\nu)-2 m] \frac{4(1-\rho)}{\nu(\nu-2)} .\right. \\
F\left(1,3-\frac{\nu}{2}, \frac{\nu}{2}+1 ; 1-\rho\right)+[m-(i \gamma \cdot p+m)] \frac{(8-4 \nu)(1-\rho)}{\nu(\nu+2)} . \\
\left.F\left(1,3-\frac{\nu}{2}, \frac{\nu}{2}+2 ; 1-\rho\right)\right\} \Gamma\left(3-\frac{\nu}{2}\right) .
\end{gathered}
$$

This decomposition is consistent, since both the self energy and its finite part depend linearly on $i \gamma \cdot p+m$. Note that $\Sigma_{f}(p . \nu)$ can be re-written as

$$
\Sigma_{f}(p, \nu)=\Sigma_{f 1}(p, \nu)+(i \gamma \cdot p+m) \Sigma_{f 2}(p, \nu),
$$

with

$$
\begin{gathered}
\Sigma_{f 1}(p, \nu)=-\frac{e^{2}}{(4 \pi)^{\frac{\nu}{2}}} m^{\nu-3}\left\{\frac{8(1-\rho)}{\nu(\nu-2)} .\right. \\
F\left(1,3-\frac{\nu}{2}, \frac{\nu}{2}+1 ; 1-\rho\right)-\frac{(8-4 \nu)(1-\rho)}{\nu(\nu+2)} . \\
\left.F\left(1,3-\frac{\nu}{2}, \frac{\nu}{2}+2 ; 1-\rho\right)\right\} \Gamma\left(3-\frac{\nu}{2}\right), \\
\Sigma_{f 2}(p, \nu)=\frac{e^{2}}{(4 \pi)^{\frac{\nu}{2}}} m^{\nu-4}\left\{(2-\nu) \frac{4(1-\rho)}{\nu(\nu-2)} .\right. \\
F\left(1,3-\frac{\nu}{2}, \frac{\nu}{2}+1 ; 1-\rho\right)-\frac{(8-4 \nu)(1-\rho)}{\nu(\nu+2)} . \\
\left.F\left(1,3-\frac{\nu}{2}, \frac{\nu}{2}+2 ; 1-\rho\right)\right\} \Gamma\left(3-\frac{\nu}{2}\right) .
\end{gathered}
$$

Note that $\Sigma_{f 1}(p, \nu)$ and $\Sigma_{f 2}(p, \nu)$ are independent of $i \gamma \cdot p+m$. Although this result seems to be correct, to obtain the true convolution we must perform the pertinent Laurent's expansion. To such an end we define

$$
f(\nu)=\frac{m^{\nu-4}}{(4 \pi)^{\frac{\nu}{2}}}\left[\left(\frac{4}{\nu-2}-\frac{2 \nu-4}{\nu}\right) m-\frac{4}{\nu}(i \gamma \cdot p+m)\right] .
$$


We then use the following expression for the gamma function

$$
\Gamma\left(2-\frac{\nu}{2}\right)=-\frac{2}{\nu-4}-C+\sum_{k=1}^{\infty} c_{k}(\nu-4)^{k}
$$

and thus obtain the corresponding Laurent expansion

$$
\begin{gathered}
f(\nu) \Gamma\left(2-\frac{\nu}{2}\right)=\frac{i \gamma \cdot p}{(4 \pi)^{2}(\nu-4)}+ \\
\frac{1}{(4 \pi)^{2}}\left\{i \gamma \cdot p\left[C+2 \ln (m)-\ln (4 \pi)-\frac{1}{2}\right]+2 m+\sum_{k=1}^{\infty} b_{k}(\nu-4)^{k}\right\} .
\end{gathered}
$$

Using the previous result, we have the following expression for the self energy

$$
\begin{gathered}
\Sigma(\nu, p)=\frac{e^{2}}{(4 \pi)^{2}}\left\{\frac{i \gamma \cdot p}{\nu-4}+\right. \\
\left.i \gamma \cdot p\left[C+2 \ln (m)-\ln (4 \pi)-\frac{1}{2}\right]+2 m+\sum_{k=1}^{\infty} b_{k}(\nu-4)^{k}\right\}+ \\
\frac{e^{2}}{(4 \pi)^{\frac{\nu}{2}}}\left\{[(i \gamma \cdot p+m)(2-\nu)-2 m] \frac{4(1-\rho)}{\nu(\nu-2)} .\right. \\
F\left(1,3-\frac{\nu}{2}, \frac{\nu}{2}+1 ; 1-\rho\right)+[m-(i \gamma \cdot p+m)] \frac{(8-4 \nu)(1-\rho)}{\nu(\nu+2)} . \\
\left.\left.F\left(1,3-\frac{\nu}{2}, \frac{\nu}{2}+2 ; 1-\rho\right)\right\} \Gamma\left(3-\frac{\nu}{2}\right)\right\}
\end{gathered}
$$

Completing the Laurent expansion we have

$$
\begin{gathered}
\Sigma(\nu, p)=\frac{e^{2}}{(4 \pi)^{2}}\left\{\frac{i \gamma \cdot p}{\nu-4}+\right. \\
i \gamma \cdot p\left[C+2 \ln (m)-\ln (4 \pi)-\frac{1}{2}\right]+2 m+\sum_{k=1}^{\infty} b_{k}(\nu-4)^{k}+ \\
\frac{e^{2}}{(4 \pi)^{\frac{\nu}{2}}} i \gamma \cdot p(1-\rho)\left[\frac{1}{3} F(1,1,4 ; 1-\rho)+\right. \\
F(1,1,3 ; 1-\rho)]+2 m(1-\rho) F(1,1,3 ; 1-\rho)+ \\
\left.\sum_{k=1}^{\infty} c_{k}(\nu-4)^{k}\right\}
\end{gathered}
$$

or equivalently

$$
\begin{gathered}
\Sigma(\nu, p)=\frac{e^{2}}{(4 \pi)^{2}}\left\{\frac{i \gamma \cdot p}{\nu-4}+\right. \\
i \gamma \cdot p\left[C+2 \ln (m)-\ln (4 \pi)-\frac{1}{2}\right]+2 m+ \\
\frac{e^{2}}{(4 \pi)^{\frac{\nu}{2}}} i \gamma \cdot p(1-\rho)\left[\frac{1}{3} F(1,1,4 ; 1-\rho)+\right. \\
F(1,1,3 ; 1-\rho)]+2 m(1-\rho) F(1,1,3 ; 1-\rho)+
\end{gathered}
$$




$$
\left.\sum_{k=1}^{\infty} a_{k}(\nu-4)^{k}\right\} .
$$

This last result can be recast as

$$
\begin{gathered}
\Sigma(\nu, p)=\frac{e^{2}}{(4 \pi)^{2}}\left\{\frac{i \gamma \cdot p+m}{\nu-4}-\frac{m}{\nu-4}+\right. \\
i \gamma \cdot p\left[C+2 \ln (m)-\ln (4 \pi)-\frac{1}{2}\right]+2 m+ \\
\frac{e^{2}}{(4 \pi)^{\frac{\nu}{2}}} i \gamma \cdot p(1-\rho)\left[\frac{1}{3} F(1,1,4 ; 1-\rho)+\right. \\
F(1,1,3 ; 1-\rho)]+2 m(1-\rho) F(1,1,3 ; 1-\rho)+ \\
\left.\sum_{k=1}^{\infty} a_{k}(\nu-4)^{k}\right\} .
\end{gathered}
$$

We are now allowed to effect then the following decomposition

$$
\Sigma(p, \nu)=A+(i \gamma \cdot p+m) B+\Sigma_{f}(p, \nu),
$$

where the constant $A$ and $B$ are given by

$$
\begin{gathered}
A=-\frac{e^{2} m}{(4 \pi)^{2}(\nu-4)}, \\
B=\frac{e^{2}}{(4 \pi)^{2}(\nu-4)},
\end{gathered}
$$

and the finite part of the self energy is

$$
\begin{gathered}
\Sigma_{f}(\nu, p)=\frac{e^{2}}{(4 \pi)^{2}}\left\{i \gamma \cdot p\left[C+2 \ln (m)-\ln (4 \pi)-\frac{1}{2}\right]+2 m+\right. \\
\frac{e^{2}}{(4 \pi)^{\frac{\nu}{2}}} i \gamma \cdot p(1-\rho)\left[\frac{1}{3} F(1,1,4 ; 1-\rho)+\right. \\
\left.\frac{1}{2} F(1,1,3 ; 1-\rho)\right]+2 m(1-\rho) F(1,1,3 ; 1-\rho)+ \\
\left.\sum_{k=1}^{\infty} a_{k}(\nu-4)^{k}\right\} .
\end{gathered}
$$

We have then the four dimensional result (the sub-index $P$ below means "physical")

$$
\begin{gathered}
\Sigma_{P}(p)=\Sigma_{f}(p, 4)=\frac{e^{2}}{(4 \pi)^{2}}\left\{i \gamma \cdot p\left[C+2 \ln (m)-\ln (4 \pi)-\frac{1}{2}\right]+2 m+\right. \\
i \gamma \cdot p(1-\rho)\left[\frac{1}{3} F(1,1,4.1-\rho)+\frac{1}{2} F(1,1,3,1-\rho)\right]- \\
2 m(1-\rho) F(1,1,3,1-\rho)\},
\end{gathered}
$$

which is the exact result of the convolution (see [1]). We arrive thus to the conclusion that $\Sigma_{P}(p, \nu)$ is the true physical self energy, a rather nice conclusion. 


\subsection{Vacuum Polarization Evaluation}

The vacuum polarization can be written in the form

$\Pi_{\mu v}(k, \nu)=\frac{e^{2}}{(4 \pi)^{\frac{\nu}{2}}} \frac{d(\nu)}{3} \Gamma\left(2-\frac{\nu}{2}\right) m^{\nu-4}\left(k_{\mu} k_{v}-\eta_{\mu v} k^{2}\right)+\Pi_{\mu v F}(k, \nu)$,

where $\Pi_{\mu v F}(k, \nu)$ is given by (2.2.5). To make the Laurent expansion we define

$$
f(\nu)=\frac{1}{(4 \pi)^{\frac{\nu}{2}}} \frac{d(\nu)}{3} m^{\nu-4}\left(k_{\mu} k_{v}-\eta_{\mu v} k^{2}\right) .
$$

Thus, we obtain

$$
\begin{aligned}
& f(\nu) \Gamma\left(2-\frac{\nu}{2}\right)=-\frac{1}{(4 \pi)^{2}}\left\{\frac{d(\nu)}{3} \frac{k_{\mu} k_{v}-\eta_{\mu v} k^{2}}{4-\nu}+\left(k_{\mu} k_{v}-\eta_{\mu v} k^{2}\right) .\right. \\
& \left.\left\{\frac{d(4)}{3}[2 \ln (m)-\ln (4 \pi)-C]+\frac{d^{\prime}(4)}{3}\right\}+\sum_{k=1}^{\infty} b_{k}(\nu-4)^{k}\right\} .
\end{aligned}
$$

Using this result, we obtain for the vacuum polarization

$$
\begin{gathered}
\Pi_{\mu v}(k, \nu)=-\frac{e^{2}}{(4 \pi)^{2}} \frac{d(\nu)}{3} \frac{k_{\mu} k_{v}-\eta_{\mu v} k^{2}}{4-\nu}-\frac{e^{2}}{(4 \pi)^{2}}\left\{\left(k_{\mu} k_{v}-\eta_{\mu v} k^{2}\right) .\right. \\
\left.\left\{\frac{d(4)}{3}[2 \ln (m)-\ln (4 \pi)-C]+\frac{d^{\prime}(4)}{3}\right\}+\sum_{k=1}^{\infty} b_{k}(\nu-4)^{k}\right\}+\Pi_{\mu v F}(k, \nu) .
\end{gathered}
$$

The finite part is then

$$
\begin{gathered}
\Pi_{\mu v f}(k, \nu)=-\frac{e^{2}}{(4 \pi)^{2}}\left\{\left(k_{\mu} k_{v}-\eta_{\mu v} k^{2}\right) .\right. \\
\left.\left\{\frac{d(4)}{3}(2 \ln (m)-\ln (4 \pi)-C]+\frac{d^{\prime}(4)}{3}\right\}\right\}+\Pi_{\mu v F}(k, 4)+\sum_{k=1}^{\infty} a_{k}(\nu-4)^{k} .
\end{gathered}
$$

Consequently, we have for the convolution (in four dimensions) the result

$$
\begin{array}{r}
\Pi_{\mu v P}(k)=\Pi_{\mu v f}(k, 4)=-\frac{e^{2}}{(4 \pi)^{2}}\left\{\left(k_{\mu} k_{v}-\eta_{\mu v} k^{2}\right) .\right. \\
\left.\left\{\frac{d(4)}{3}[2 \ln (m)-\ln (4 \pi)-C]+\frac{d^{\prime}(4)}{3}\right\}\right\}+\Pi_{\mu v F}(k, 4) .
\end{array}
$$

\section{Discussion}

We used the new QFT-paradigm based on STEDELI-distributions that overcomes the old problem of QFT-infinities. With this new paradigm, we have here solved, for instance, the non renormalizable problem posed by $\lambda \frac{\phi^{4}}{4 !}$ theory in six dimensions. The old QFTparadigm can do nothing here. Instead, we solved it.

Using STEDELI, we were thus able here to successfully handle in novel fashion several old QFTproblems. 
It is of the essence to realize that, in QFT, when one uses perturbative expansions, we are dealing with products of distributions in configuration space or, analogously, with convolutions of distributions in momentum space.

Using such convolution, we have exactly evaluated, for the first time, the electron self energy and the vacuum polarization in QED.

\section{Conflicts of Interest}

The authors declare no conflicts of interest regarding the publication of this paper.

\section{References}

[1] Plastino, A. and Rocca, M.C. (2018) Quantum Field Theory, Feynman-, Wheeler Propagators, Dimensional Regularization in Configuration Space and Convolution of Lorentz Invariant Tempered Distributions. Journal of Physics Communications, 2, Article ID: 115029. https://doi.org/10.1088/2399-6528/aaf186

[2] Bollini, C.G., Escobar, T. and Rocca, M.C. (1999) Convolution of Ultradistributions and Field Theory. International Journal of Theoretical Physics, 38, 2315-2332. https://doi.org/10.1023/A:1026623718239

[3] Bollini, C.G. and Rocca, M.C. (2004) Convolution of Lorentz Invariant Ultradistributions and Field Theory. International Journal of Theoretical Physics, 43, 1019-1051. https://doi.org/10.1023/B:IJTP.0000048599.21501.93

[4] Bollini, C.G. and Rocca, M.C. (2004) Convolution of $n$ Dimensional Tempered Ultradistributions and Field Theory. International Journal of Theoretical Physics, 43, 59-76. https://doi.org/10.1023/B:IJTP.0000028850.35090.24

[5] Bollini, C.G., Marchiano, P. and Rocca, M.C. (2007) Convolution of Ultradistributions, Field Theory, Lorentz Invariance and Resonances. International Journal of Theoretical Physics, 46, 30303059. https://doi.org/10.1007/s10773-007-9418-y

[6] Plastino, A. and Rocca, M.C. (2020) Non-Relativistic Quantum Field Theory of Verlindes Emergent Entropic Gravity. Annals of Physics, 412, Article ID: 168013. https://doi.org/10.1016/j.aop.2019.168013

[7] Sebastiao e Silva, J. (1958) Les fonctions analytiques comme ultra-distributions dans le calcul oprationnel. Mathematische Annalen, 136, 38-96. https://doi.org/10.1007/BF01350287

[8] Schwartz, L. (1966) Théorie des distributions. Hermann, Paris.

[9] Bollini, C.G. and Giambiagi, J.J. (1972) Lowest Order "Divergent" Graphs in $v$-Dimensional Space. Physics Letters B, 40, 566568. https://doi.org/10.1016/0370-2693(72)90483-2

[10] Bollini, C.G. and Giambiagi, J.J. (1972) Dimensional Renorinalization: The Number of Dimensions as a Regularizing Parameter. Il Nuovo Cimento B, 12, 20-26. 
[11] Bollini, C.G. and Giambiagi, J.J. (1996) Dimensional Regularization in Configuration Space. Physical Review D, 53, 5761. https://doi.org/10.1103/PhysRevD.53.5761

[12] Plastino, A. and Rocca, M.C. (2020) Gupta-Feynman based Quantum Field Theory of Einstein's Gravity. Journal of Physics Communications, 4, Article ID: 035014.

https://doi.org/10.1088/2399-6528/ab8178

[13] Plastino, A. and Rocca, M.C. (2020) Quantization of Newtons Gravity. Journal of Modern Physics, 11, 920-927.

https://doi.org/10.4236/jmp.2020.116056

[14] Zamora, D.J., Rocca, M.C., Plastino, A. and Ferri, G.L. (2018) Dimensionally Regularized Boltzmann-Gibbs Statistical Mechanics and Two-Body Newtons Gravitation. Physica A: Statistical Mechanics and Its Applications, 503, 793-799.

https://doi.org/10.1016/j.physa.2018.03.019

[15] Zamora, D.J., Rocca, M.C., Plastino, A. and Ferri, G.L. (2018) Dimensionally Regularized Tsallis Statistical Mechanics and Two-Body Newtons Gravitation. Physica A: Statistical Mechanics and Its Applications, 497, 310-318. https://doi.org/10.1016/j.physa.2018.01.018

[16] Gel'fand, I.M. and Shilov, G.E. (1964) Generalized Functions, Vol. 1. Academic Press, Cambridge, MA.

[17] Gradshteyn, I.S. and Ryzhik, I.M. (1980) Table of Integrals, Series and Products. Academic Press, Cambridge, MA. 